\title{
The Cost Minimization Model in Warehouse Distribution System
}

\author{
Syed Abdul Rehman Khan, Yu Zhang* \\ School of Economics and Management, Chang'an University, Xi'an, China
}

Email address:

sber.linda18@gmail.com (Yu Zhang)

${ }^{*}$ Corresponding author

To cite this article:

Syed Abdul Rehman Khan, Yu Zhang. The Cost Minimization Model in Warehouse Distribution System. International Journal of Management and Fuzzy Systems. Vol. 3, No. 5, 2017, pp. 75-79. doi: 10.11648/j.ijmfs.20170305.13

Received: August 13,2017; Accepted: August 31, 2017; Published: November 8, 2017

\begin{abstract}
In today's world, warehousing and distribution centres play a vital role to fulfil customer demand and requirement. Firms cannot ignore the importance of warehouse and distribution centres. In addition, firms are using DCs (distribution centres) and warehouses for valuation addition purposes such as, postponement strategies, break-bulking, consolidation, and packing and packaging etc. This research aim is to develop a model to reduce warehouse cost and increase or improved customer service. The results and discussions with personnel's of distribution department seem to offer a lesson. It is that, though human distribution "optimizers" out of necessity, must concentrate their efforts on maintaining the most economical primary shipment transportation system, a mechanical "optimizer" like as MODS (model of optimizing distribution centres) is able to find out and determine the most economical system from the standpoint of whole the relevant costs to be considered.
\end{abstract}

Keywords: Warehousing, Distribution Centres, MODS, Transportation Routes

\section{Introduction}

In this research paper, mainly four elements are considered are warehouses, demand points, manufacturing plants, and transportation routes. Therefore, a solution to that problem would give answers to questions like:

a. Where should the warehouses and manufacturing area be located?

b. What Capacity should they have?

c. What modes and routes should be used?

d. What quantity or size should and how frequently should shipments?

That problem is transhipment, some kind of what is known in the literature as the problem of the transportation-location. Ref. [1-3] to solve this critical problem, we have developed a model "Optimizing" distribution systems (MODS). Ref. [4] the three main methodological categories are simulation, heuristic, and exact algorithm, for treating such problems. MODS follow the Heuristic approach, which is heuristic. In specifically, it represents an extension of the first heuristic approach to this problem, which was explained by Kuehn \&
Hamburger [2].

MODS approach was developed for defining and determining the optimal solution. An organization, which distributes their products in the Unites States; at the time of this study was begun, this organizations distributed six different product categories through a system of 13 (thirteen) public warehouses. And each category of product manufactured at single site.

The specific questions the organization wished answered the following;

a. How many warehouses they should need to use?

b. And on which location, these warehouses should be located?

c. Which demand points should be serviced from each warehouse?

Furthermore, it was defined that, organization not only interested in defining an optimal system by providing answers all of those questions, but they also interested a model should be capable to determining the optimal distribution pattern for a specified set of warehouses and evaluating the performance of a particular distribution system. In order, to keep problem in reasonable limitation 
and size, one question addressed was what is a point of demand? A research study indicated that the large number of individual cities served made it not feasible to treat everyone as a single point of demand. One important deciding factor in this selection was the fact that the organization's systematic freight records included or incorporated a code of county (In the US) for every customer.

An analysis of those data of demand performed to determine which of the county demand points had to be treated in the research in order to control and maintain a reasonable representation of the national distribution. As per this analysis result showed that, more than 3000 countries and counties are equivalents in the contiguous US only almost 1230 received freight shipments. However, in this analysis showed that, $90 \%$ of the total, was accounted by 225 countries, $95 \%$ by 350 and $99 \%$ is by 650 . On the basis of primarily on that results it decided to consider the 225 countries which are accounted for $90 \%$ of the total shipments (secondary) as per the set of demand points for the research study. At one point, the total demand was considered to develop (the main city within the country), meanwhile mostly countries have one large and big metropolitan city, this approximation seemed reasonable. The further limitation placed in the list of potential warehouse site to treat. Based on the formerly and previously showed analysis of freight shipments, on cost and geographic considerations, the study team, which consisted of operations research personnel and distribution department, defined a set almost 40 warehouses' points to be considered. These are all metropolitan cities and areas located throughout the country.

\section{MODS Model and Literature Review}

In the model development, one consideration was that criterion of optimality is suitable for this problem. The research team approached that question through identifying the purpose of having regional warehouses. Actually, three aspects to this purpose:

a. The reduction in the cost of transportation due to direct shipment to demand points through permitting quantity primary shipments. (e.g. manufacturing facility to warehouses)

b. The reduction in the cost of delivery by combining items, products manufactured at different points into single final / secondary shipments. (e.g. warehouses to demand points)

c. Improvement in the customers' service through minimizing the delivery time relative to direct shipment to points of demand.

Accordingly, the model should explicitly treat both customer service level and cost in defining a distribution system (optimal). Actually it is usually not possible to optimize two different, conflicting goals such as these; it was decided to try to develop a model, which can minimize cost for a particular minimum service level for all points of demand. Actually this was the sense of optimal used hereafter. One of the further consideration was an identification of cost, which are relevant in the defining a distribution system (optimal). Although primarily cost studies mentioned; the primary cost (transportation, secondary cost and inter-warehouse shipment accounted for the majority of distribution costs, including considered other costs. MODS was developed particularly for the handling costs, and storage, inventory carried, income and franchise taxes, administrative cost, and order processing transmission equipment.

In researches including freight shipments, the rate of transportation structure usually shows a problem. The basic reason is that the rate used for a route depends on quantity shipped, transportation' modes. While this research was restricted to over ground transportation, it is still compulsory to consider following;

a. FTL (full truck load) rates

b. LTL (less than truck) rates

c. Full freight car load rates

d. Car load rates with a penalty

A rate model was developed for the primary \& interwarehouse routes, which needs two full-load rates and one less than truck load (FTL) for every route. Further analysis showed that all shipments (secondary) could be considered to be made at less than truck load (LTL) rates.

Due to the particular need for a model of cost minimization, the literatures and previous researches were searched and read, which could satisfy the elements of that problem. Ref. [6-10] mentioned that this problem of distribution differs from those, which found in the literature reviews. Specifically, the problem is includes; mixed product shipments, multi products; fixed warehouse cost, rates of transportation with economies of scale and customer service level. A major reason for considering of all those aspects is the need to develop the confidence of the operating personnel, who would be fully responsible for implementing the model's results.

The model can be described as heuristic programming model. Ref. [11-12] it is very good solution for the problem but may be not optimal solution. The model involves reading the rates of transportation from a magnetic tape, storing them on a magnetic tape, structuring the parameters for the rate's model. The remainder of model inputs are read from punched cards. Secondly main step in the model use the transportation algorithm to solve the problem of transhipment individually for every product categories. These results are observed in the rate adjustment to control and determine if the transportation algorithm were based on wrong, incorrect rates.

The solution, which is established in this method, is based upon variable costs, no fixed costs considered. However, in the solution, warehouses' number will be greater than the optimum. If the fixed costs are significant and the fixed cost associated with the warehouses in the solution are determined. As well the measures of level of service for this solution are computed.

The next stage is to print out a management output reports. The reports included six separate parts or reports. The first is 
a complete and detailed report of all primary and interwarehouse shipments in the solution. For every origin to destination combination with a positive shipment volume the report lists the item and product size, category, frequency, transportation rates, total cost of transportation, inventory level, warehouse handling cost, personal property tax, inventory interest cost. The second report gives almost same detail for the secondary shipments. In the third reports, the service level measures are given. In the fourth, summary of all the cost of variable for every warehouse. In the fifth reports, indicates the grand total cost of warehouse and fixed costs. And sixth report finally summarizes all the cost of variables by each product category.

The final stage is the modification elimination procedure. The starting part of that procedure searches for answer to given question. Considering both fixed and variable costs, is it economical to substitute any of the inactive (not included in the solution) warehouses for one of the active warehouses? And after any some modifications are made, the procedure next finding for an answer to a next question. If a fixed warehouses' numbers eliminated from the solution, which of the eliminations will minimize, decrease the variable cost the most? (Fixed costs is not included, answering this question) since they will be accounted for in the next iteration by the modification portion.

Due to the two points this procedure is more acceptable than other approaches of eliminations. First, basic objection to elimination procedure is "once a warehouse leaves the solution; there is no provision to accept, allow it to return towards solution. Nevertheless, the MODS approach follow to the elimination process through the modification process at the next iteration. Thus, eliminated warehouses may return towards solution, if they offer a more economical option, alternative to a warehouse.

The second point is that, in that procedure every warehouse has a list of substitute warehouses specified for it. However, in the modification procedure, it is not compulsory to consider all the non-active warehouses which are designated as substitutes for a given active warehouses must be considered as an alternative, substitution. Correspondingly, in this elimination procedure, only active warehouse which are on another active warehouse's substitute, alternative list have to be considered as a replacement. We should hasten to point out "this device can restrict the model's ability to explain, define an optimal system. So it must be used with some care.

The following modification-elimination procedures, newly defined solution is used as the particular distribution system into the algorithm of transportations. The algorithm will be used to determine the optimal distribution territory for every warehouse in this particular solution. The remainder of the process of evaluation is as before, and the complete entire process is repeated again until an increase in the total cost is originated.

\section{The Evaluations and Results}

MODS has been programmed in FORTRAN for an IBM System (360 Model 40 computer) for solving problem previously explained. 3 separate programs were written corresponding to hat three parts of figure 1 .

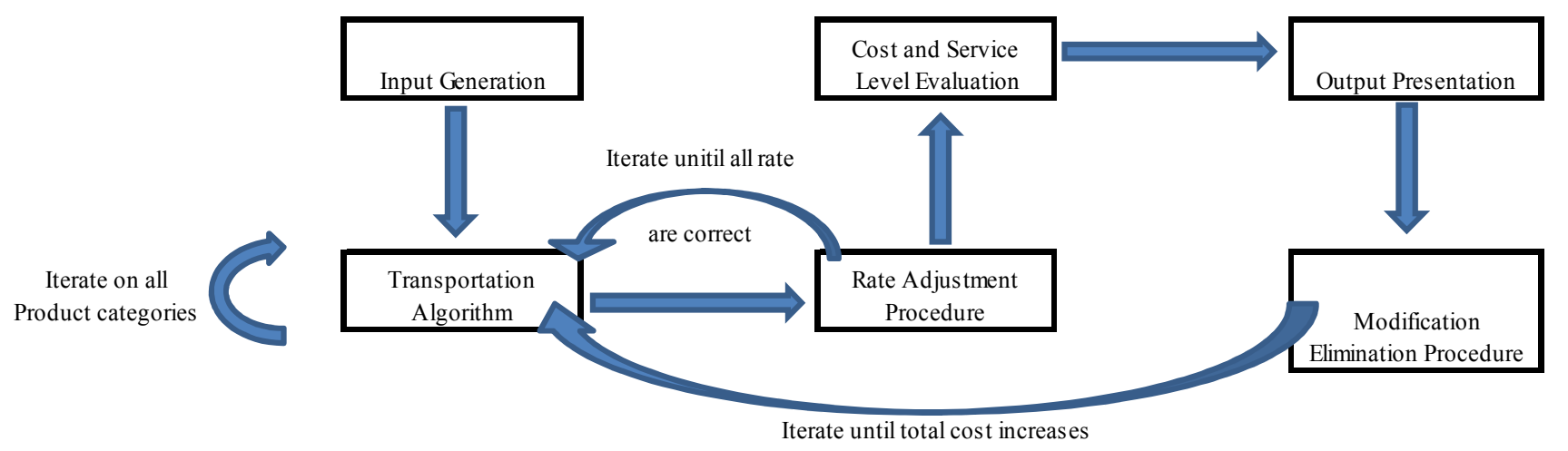

Figure 1. Model for Optimizing Distribution Systems.

The largest of those programs needs almost 170,000 bytes of core plus magnetic disk storage $\&$ magnetic tape drives. The programmed model has been checked and validated by using the real data for the seven-state area west of the Rocky Mountains.

Modes have three different modes of operations:

a. Evaluation of the service and cost

b. Optimization of warehouse

c. Determination of an optimal distribution system

The first full-country runs were option 1 runs with actual distribution system parallel at a same time. Those runs were the last stage in the validation process, while the results of the model were compared with the actual operating results. As per the result comparison few minor changes was made in parameters of the model. Due to these changes made, the output from the model in terms of cost component values nearly resembled with the results of actual cost.

The further runs made were option 2 runs with the current system. While, the one change from the last runs was that the model was used to optimize warehouse spaces, territories. And these runs resulted total cost almost $\$ 3.02$ million yearly as opposed to $\$ 3.08$ million from the previous run. While, the mean service distance increased from 158 miles to 191 miles.

On this point, distribution management had already 
decided to add a 14 warehouse into the current system. This was compulsory to alleviate, improve a severe distribution problem in the eastern US. The option 2 of MODS was used to compare the 2 possible new locations that were being careful considered and to define the warehouse areas, territories for the new system. The betterment of the 2 system had total estimated cost of $\$ 2.98$ million, and 170 miles a service distance mean.

According to the changes in the rate structure of transportation and the availability of updated demand data after the above runs, all the model data was updated as well complete series of new runs was made. 5 sets of runs were made with the new data.

These included:

a. The 13 old warehouse system

b. The 14 new warehouse system

c. The new system with optimized all territories

d. The optimal system as determined through MODS

e. A good, but suboptimal system

The summary of the results of these evaluations runs has been given in Table 1 . The shown costs are as before for a whole year's operation.

Table 1. Summary of the MODS Results for Distribution System.

\begin{tabular}{|c|c|c|c|c|c|}
\hline Characteristics & Old System & New System & Optimized New System & Optimal System & Suboptimal System \\
\hline Total Variable Costs $(\$ \times 103)$ & 3458 & 3360 & 3325 & 3054 & 3113 \\
\hline Total Fixed Costs $(\$ \times 103)$ & 130 & 139 & 139 & 207 & 187 \\
\hline Grand Total Costs $(\$ \times 103)$ & 3588 & 3499 & 3464 & 3261 & 3300 \\
\hline Service Level Mean (miles) & 174 & 181 & 194 & 100 & 120 \\
\hline Number of Field/Consolidation Warehouses & $13 / 1$ & $14 / 1$ & $14 / 1$ & $32 / 10$ & $27 / 5$ \\
\hline
\end{tabular}

Two items concerning those results need elaboration. First, only 2 of the 6 items, products categories were high volume item, products. In specifically, these 2 product categories accounted for almost $90 \%$ of the whole distribution volume. While the other 4 item categories were distributed by consolidation warehouses.

In the actual system, these all products' production was shipped to one main warehouse and then mixed shipment of these 4 products were sent to remaining all warehouses. Thus, the whole system had one warehouse of consolidation. As will be noted in Table 1, the optimal solution of MODS called for 10 consolidation warehouses.

The second main point related with the fact that the products of another division of this organization was distributed by this system. Although those items, products was not to be considered in defining the system (optimal). Cost studies shown that any savings originate for the 6 item categories considered would be $50 \%$ increase in actuality as a result of the additional products. Due to this fact, the total forecasted savings in shifting from the old system towards new system (optimal system) is almost one and half a million dollars yearly.

As was shown in Table 1, the warehouses' number in the new system (optimal system) is surprisingly high. Because that a 27 warehouse system, the so-called suboptimal system, was evaluated in detail. While, the evaluations' results confirmed the conclusion " 32 warehouses are better through a cost difference (projected) estimated $\$ 59000$ yearly.

Given this the inputs used are valid; the conclusion that 32 warehouses are optimal number also seems to withstand the logic test. Namely, the total (secondary) transportation cost will be minimizing if as more and more warehouses will be utilized. Since, in generally the secondary service distances will be minimizing. In addition, if volume at every warehouse for the majority of the items is like the full-load rates are still possible for the majority of the primary shipments, the total cost of the variable will be decreased for an increased number of warehouses. On the opposite, the fixed costs make up only almost $5 \%$ of the total cost, so that one would assume the optimal numbers of warehouse to be somewhat greater than the number that was being used.

It needs to be pointed out that the solution (optimal) was not very appealing to the personnel of distribution management. The major reason for this is an at least 2 fold, first, the effort necessary to coordinate with the inventories of a larger warehouses' number increased. While, in the model this effect was reflected by the inclusion of an administration' fixed cost attached with every warehouse.

A Second objection, with large warehouses' numbers, the primary shipments for few low-volume items will have to be made in LTL (less than truck) load lots, which is expensive. Moreover, that effect will be even more predominant, prevalent in the optimal solution; meanwhile it utilizes 10 warehouses for consolidation for the low volume items instead of only one. On the opposite hand, an detailed examination of the MODS results shown that the total cost of overall distributing the low volume products by the optimal system with some LTL (less than truck) load primary rates is actually less by almost $\$ 5000$ yearly than the cost of the current practices with one warehouse for consolidation.

\section{Conclusion}

These results and discussions with personnel's of distribution department seem to offer a lesson. It is that, though human distribution "optimizers" out of necessity, must concentrate their efforts on maintaining the most economical primary shipment transportation system, a mechanical "optimizer" like as MODS is able to find out and determine the most economical system from the standpoint of whole the relevant costs to be considered. In conclusion, these efforts showed an attempt to generate a model for optimizing a real distribution system. While the model's result is more complex and cumbersome to use as compare to those which has been discussed in the literature, it does reflect many aspects of ground reality that the simpler model do not treat. 


\section{References}

[1] Atkins, Robert J. and Shriver, Richard H., (1968), "New approach to facilities location" Harvard Business Review Vol. 46, No. 03.

[2] Kuehn, Alfred A. and Hamburger, Michael J., (1963), “A Heuristic Program for Locating Warehouses," Management Science Vol. 9, No. 4.

[3] Cooper, Leon, (1972), "The transportation-location problem," Operations Research Vol. 20, No. 1.

[4] Devine, M. D. and Lesso, W. G., (1972), "Models for minimum cost development of Offshore oil fields," Management Science Vol. 18, No. 8.

[5] Feldman, E., Lehrer, F. A., and Ray, T. L., (1966), "Warehouse Location under Continuous Economies of Scale," Management Science Vol. 12, No. 9.

[6] Khumawala, Basheer M., (1972), "An Efficient Branch and Bound Algorithm for the Warehouse Location Problem," Management Science Vol. 18, No. 12.

[7] Michael, George C., (1972), "A Review of Heuristic Programming," Decision Sciences Vol. 3, No. 3.

[8] Revelle, Charles, Marks, David, and Liebman, Jon C., (1970), "An Analysis of Private and Public Sector Location Models," Management Science Vol. 16, No. 11.

[9] Whybark, D. Clay and Khumawala, Basheer M., (1972), “A Survey of Facility Location Problems," Krannert Graduate School of Industrial Administration Institute paper Series No. 350, Purdue University.

[10] Khan, S. A. R., Dong, Q. L., \& Yu, Z. (2016). Research on the Measuring Performance of Green Supply Chain Management: In the Perspective of China. International Journal of Engineering Research in Africa, 27, 167-178. https://doi.org/10.4028/www.scientific.net/JERA.27.167

[11] Khan, S. A. R., \& Qianli, D. (2017). Impact of green supply chain management practices on firms' performance: an empirical study from the perspective of Pakistan. Environmental Science and Pollution Research. https://doi.org/10.1007/s11356-017-9172-5

[12] Khan, S. A. R., Qianli, D., Song Bo, W., Zaman, K., \& Zhang, Y. (2017). Environmental logistics performance indicators affecting per capita income and sectoral growth: evidence from a panel of selected global ranked logistics countries. Environmental Science and Pollution Research, 24(2), 15181531. https://doi.org/10.1007/s11356-016-7916. 